\title{
XÁC ĐINNH HÀM LƯỢNG POLYPHENOL VÀ EGCG TRONG CHĖ, SẢN PHẨM CHÈ BẰNG PHƯƠNG PHÁP UV-VIS VÀ HPLC
}

\author{
Phạm Thị Ngọc Mai ${ }^{1}$, Lê Thái Bình¹, Phạm Huy Đông², Nguyễn Thị Tuyết Nhung, \\ Phạm Tiến Đức ${ }^{1}$, Nguyễn Thị Ánh Hường ${ }^{1^{*}}$ \\ ${ }^{1}$ Khoa Hóa học, Truòng Đại học Khoa học Tụ nhiên, Đại học Quốc gia Hà Nội \\ ${ }^{2}$ Viện Hóa học Công nghiệp Việt Nam
}

(Ngày đến tòa soạn: 15/1/2018; Ngày sủa bài sau phản biện: 23/2/2018; Ngày chấp nhận đăng: 5/3/2018)

\section{Tóm tắt}

OHÈ và các sản phẩm chè đã được biết đến

và sư dụng tù rất lâu, thậm chí trở thành nét văn hóa truyền thống ở nhiều quốc gia trên thế giới, trong đó có Việt Nam. Chất luợng chè phu thuộc vào hàm luợng các hoạt chất điển hình ví dụ nhu nhóm polyphenol và các hoạt chất của nó (EGCG, EGC, catechin,...). Do đó, viẹc nghiên cứu các phurong pháp xác định các hoạt chất này là cần thiết nhằm đánh giá chất lượng chè và sản phẩm chè. Trong nghiên cúu này, phwơng pháp UV-Vis được áp dụng để xác định polyphenol tổng và phưong pháp HPLC dùng để xác định hàm lượng hoạt chất chính trong nhóm polyphenol là EGCG. Giới hạn phát hiện đạt được là 0,12 $\mathrm{mg} / \mathrm{g}$ và $0,064 \mathrm{mg} / \mathrm{g}$ tương úng với polyphenol tổng và EGCG. Các phương pháp đã được áp dụng để xác định hàm luợng EGCG và polyphenol tổng trong 10 mẫu chè và sản phẩm chè. Kết quả cho thấy hàm lương các chất này khá dao động trong các mẫu khác nhau, tuy nhiên lại có mối liên quan nhất định giữa hàm lương EGCG và polyphenol tổng trong tùng mẫu chè.

Tù khóa: polyphenol, ECCG, chè, UV-Vis, HPLC

\section{1. ĐĂT VẤN ĐỀ}

Chè (trà) là loại thức uống lâu đời và được sử dụng rộng rãi ở Việt Nam cũng như trên thế giới. Nhiều nghiên cứu đã chứng minh, uống chè có tác dụng phòng ngừa và chữa trị nhiều loại bệnh khác nhau như các bệnh về tim mạch và ung thư. Ngày nay, việc sản xuất, sử dụng chè và các thành phần chiết xuất từ chè ngày càng phát triển. Một trong các thành phần được quan tâm và quyết định chất lượng chè là polyphenol. Chất này có hoạt tính chống oxy hóa mạnh và chiếm hàm lượng lớn trong lá chè, nên được sử dụng với nhiều mục đích trong các ngành công nghiệp thực phẩm, dược phẩm, mỹ phẩm $[1,2,3,4]$.

Thành phần polyphenol trong lá chè rất đa dạng, nhưng gồm chủ yếu là các flavonoid và tanin. Các polyphenol này chiếm khoảng 13-35\% trọng lượng chè khô. Trong lá chè xanh, các thành phần chủ yếu là catechin và các dẫn xuất của catechin (như: catechin, epigallocatechin (EGC), gallocatechin (GC), epigallocatechin gallat (EGCG), epicatechin (EC), epicatechin gallat (ECG)...), các flavonol (Quercetin, Kaempferol, Myricetin) và các dẫn xuất khác của flavonol, theaflavin và diosmin (theaflavin) là sản phẩm của sự oxy hóa đồng thời ECG và EGCG [5]. Trong đó, EGCG được biết đến là hoạt chất chính, quan trọng ảnh hưởng trực tiếp đển chất lượng chè và các sản phẩm chè.

Từ các phân tích nêu trên có thể nhận thấy, việc xác định hàm lượng polyphenol tổng và EGCG trong chè có vai trò quan trọng trong việc đánh giá chất lượng và các tác dụng sinh dược học của sản phẩm chè. Các phương pháp thường được sử dụng để xác định tổng và các dạng polyphenol trong chè gồm: phương pháp quang phổ hấp thụ phân tử (UV-Vis), phương pháp sắc ký lỏng hiệu năng cao (HPLC), phương pháp điện di mao quản,...[2, 3, 5, 6]. Trong nghiên cứu này, phương pháp UV-Vis có ưu điểm đơn giản, hiệu quả, được sử dụng để nghiên cứu xác định hàm lượng polyphenol tổng, phương pháp HPLC với

\footnotetext{
*Điện thoại:0946593969 Email:nguyenthianhhuong@hus.edu.vn
}

8 | Tạp chí KIỂM NGHIỆM VÀ AN TOÀN THỰC PHẨM (Số1-2018) 
khả năng phân tách và độ tin cậy cao được sử dụng để nghiên cứu định lượng thành phần EGCG trong chè.

\section{2. ĐỐI TƯợNG VÀ PHƯƠNG PHÁP NGHIÊN CÚU}

\subsection{Hóa chất, thiết bị}

Các chất chuẩn: acid gallicmonohydrat, epigallocatechin gallat (Sigma-Aldrich) [2, 3]. Các hóa chất tinh khiết phân tích: natri hydroxyd $(\mathrm{NaOH})$, thuốc thử Folin - Ciocalteu, natri carbonat $\left(\mathrm{Na}_{2} \mathrm{CO}_{3}\right)$, methanol, acid pecloric (Merck), nước cất 2 lần.

Dung dịch chuẩn gốc acid gallic, tương đương với acid gallic khan nồng độ khoảng 1000 ppm: Cân chính xác khoảng $0,11 \mathrm{~g}$ acid gallic monohydrat $(\mathrm{M}=188,14)$, hòa tan vào bình định mức $100 \mathrm{~mL}$, định mức đến vạch bằng nước cất và lắc đều. Dung dịch được bảo quản ở ngăn mát tủ lạnh.

Dung dịch chuẩn EGCG: cân chính xác khoảng $0,45 \mathrm{~g}$ chất chuẩn EGCG, hòa tan vào bình định mức $100 \mathrm{ml}$ và định mức bằng methanol đến vạch, lắc đều được dung dịch chuẩn EGCG 4500 ppm.

Thiết bị quang phổ hấp thụ nguyên tử (UVVis) model- 2401, Hãng Shimadzu, Nhật Bản.

Thiết bị sắc ký lỏng HPLC của Hãng Shimadzu (LC20AD) với detector PDA, cột sắc kí Supelco $\mathrm{C} 18(250 \mathrm{~mm} \times 4,6 \mathrm{~mm} \times 5 \mu \mathrm{m})$ và tiền cột tương ứng.

\subsection{Xử lý mẫu}

Mẫu nghiên cứu: một số loại chè và sản phẩm từ chè (chè khô, trà túi lọc, chè nhập khẩu...) mua ngẫu nhiên trên thị trường Hà Nội.

Phương pháp xử lý mẫu phân tích tổng hàm lượng polyphenol bằng UV-Vis: Cân chính xác khoảng $0,2 \mathrm{~g}$ mẫu đã đồng nhất vào cốc $100 \mathrm{~mL}$. Thêm $25 \mathrm{~mL}$ dung dịch chiết methanol: nước $(70: 30, v: v)$, lắc đều bằng votex, rung siêu âm ở $70^{\circ} \mathrm{C}$ trong 30 phút rồi ly tâm (6000 vòng/phút) trong 5 phút. Thu dịch chiết và tiến hành chiết lặp phần bã bằng $20 \mathrm{~mL}$ dịch chiết. Gộp dịch chiết rồi định mức thành $50 \mathrm{~mL}$ bằng dịch chiết. Lọc trên giấy lọc băng xanh. Lấy chính xác $0,5 \mathrm{~mL}$ dịch chiết, thêm 2,5 mL thuốc thử Folin - Ciocalteu $10 \%$ và $2 \mathrm{~mL} \mathrm{Na}_{2} \mathrm{CO}_{3} 7,5 \%$, trộn đều, để yên 60 phút trước khi phân tích trên thiết bị UV-Vis.

Phương pháp xử lý mẫu phân tích hàm lượng EGCG bằng HPLC: Cân chính xác khoảng $0,2 \div 0,5 \mathrm{~g}$ mẫu đã đồng nhất vào cốc $100 \mathrm{~mL}$. Thêm $25 \mathrm{~mL}$ methanol, lắc đều bằng votex trong 1 phút, rung siêu âm ở $70^{\circ} \mathrm{C}$ trong 30 phút rồi ly tâm (6000 vòng/phút) trong 5 phút. Thu dịch chiết và tiến hành chiết lặp phần bã bằng $20 \mathrm{~mL}$ methanol. Gộp dịch chiết rồi định mức thành 50 $\mathrm{mL}$ bằng methanol. Lọc qua giấy lọc và màng lọc $0,2 \mu \mathrm{m}$ trước khi phân tích trên hệ thống HPLC.

3. KẾT QUẢ VÀ BÀN LUẬN

\subsection{Nghiên cứu xác định hàm lượng tổng} polyphenol trong chè bằng phương pháp UV-Vis 3.1.1. Khảo sát lựa chọn bước sóng cưc đại ( $\lambda_{\text {max }}$ ) và độ đặc hiệu

Phổ hấp thụ phân tử của phức tạo bởi dung dịch chuẩn acid gallic 50 ppm và thuốc thử FolinCiocalteu được khảo sát trong khoảng bước sóng từ $700 \div 800 \mathrm{~nm}$ (Hình 1$)$. Từ đó, xác định được giá trị độ hấp thụ cực đại ( $\lambda$ max) của acid gallic là ở bước sóng $737 \mathrm{~nm}$. Bước sóng này được sử dụng cho các nghiên cứu xác định polyphenol tổng bằng phương pháp UV-Vis.

Độ đặc hiệu của việc xác định polyphenol

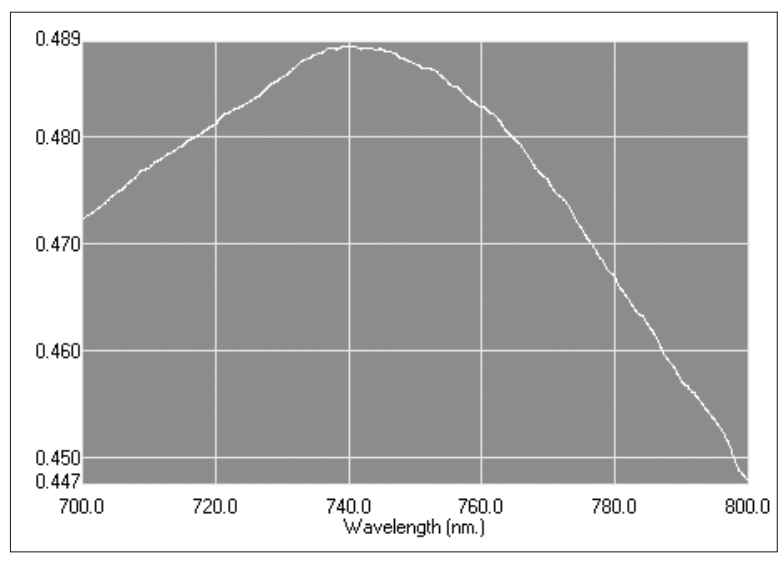

Hình 1. Phổ hấp thụ của dung dịch chuẩn acidgallic 50 ppm

ở bước sóng này cũng được kiểm chứng với mẫu trắng, mẫu thực tế (mẫu chè khô) và mẫu thực thêm chuẩn. Kết quả cho thấy mẫu trắng hầu như không cho tín hiệu ở bước sóng này, mẫu chè khô và mẫu chè khô thêm chuẩn đều cho tín hiệu với giá trị đạt cực đại tại $737 \mathrm{~nm}$. Điều này cho thấy phương pháp có độ đặc hiệu tốt.

\subsubsection{Khảo sát dung môi chiết, thời gian và nhiệt} độ chiết

Mức độ hòa tan của một chất trong các hệ dung môi khác nhau là khác nhau. Do đó việc lựa Tạp chí KIË̉M NGHIỆM VÀ AN TOÀN THỰC PHẨM (Số1-2018) | 9 
chọn hệ dung môi nhằm chiết được tối đa chất phân tích ra khỏi nền mẫu là rất quan trọng. Qua tham khảo tài liệu [6] cho thấy, các dung môi chiết hiệu quả polyphenol từ nền mẫu thực vật gồm: methanol, aceton, nước, ethyl acetat và diethyl ete. Trong nghiên cứu này, chúng tôi lựa chọn hệ dung môi methanol : nước với các tỉ lệ được khảo sát là $60: 40,70: 30,80: 20$ và $100 \%$ methanol. Kết quả khảo sát được chỉ ra trong Hình 2.

Kết quả cho thấy, hàm lượng tổng polyphenol

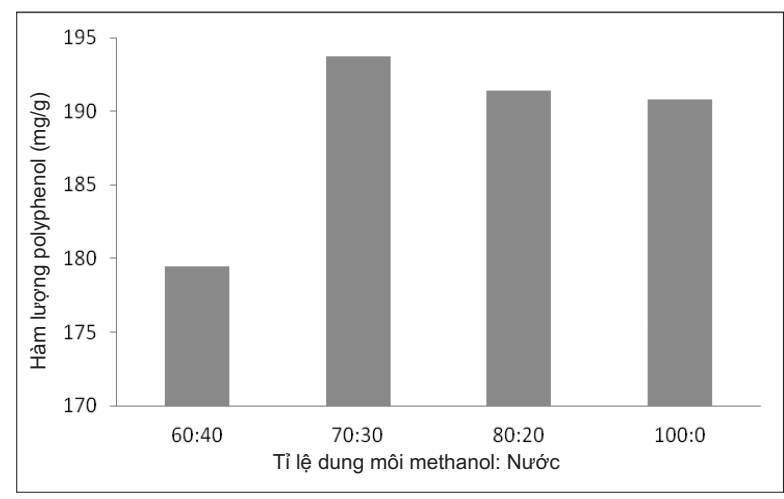

Hình 2. Kết quả khảo sát tỉ lệ dung môi chiết polyphenol trong mẫu chè

thu được khi chiết ở tỉ lệ dung môi metanol: nước $=70: 30,80: 20$ và $100 \%$ methanol không khác nhau nhiều nhưng cao hơn khi sử dụng tỉ lệ 60:40. Để tiết kiệm dung môi, chúng tôi lựa chọn tỉ lệ dung môi chiết tối ưu methanol : nước là 70:30.

Kết quả khảo sát các thời gian chiết 30,60 , 90, 150 phút cho thấy: ở thời gian chiết 30 phút thì cho kết quả hàm lượng thấp nhất, khi tăng thời gian chiết lên 60,90 phút thì độ thu hồi thay đổi không đáng kể và đều cho hàm lượng polyphenol cao. Khi tăng thời gian chiết lên 150 phút, hàm lượng polyphenol bị giảm đi đáng kể, do hiệu quả chiết giảm khi dung môi methanol bị bay hơi nhanh ở nhiệt độ cao. Do đó, chúng tôi chọn thời gian chiết 60 phút cho các khảo sát tiếp theo.

Kết quả khảo sát nhiệt độ chiết $50^{\circ} \mathrm{C}, 60^{\circ} \mathrm{C}$, $70^{\circ} \mathrm{Cvà} 80^{\circ} \mathrm{C}$ cho thấy, trong khoảng từ $50^{\circ} \mathrm{C}$ đến $70^{\circ} \mathrm{C}$ hiệu quả chiết tăng lên, tuy nhiên khi nhiệt độ tăng lên $80^{\circ} \mathrm{C}$ thì hàm lượng polyphenol thu được giảm đi đáng kể, có thể do quá trình thủy phân, phản ứng oxy hóa khử hoặc dung môi bị bay hơi. Do đó, nhiệt độ chiết là $70^{\circ} \mathrm{C}$ được lựa chọn nhằm đạt được hiệu quả tốt nhất.

3.1.3. Đánh giá phưong pháp UV-Vis xác định

\section{polyphenol tổng}

Các nội dung đánh giá phương pháp gồm: xây dựng đường chuẩn, độ chụm (thông qua độ lặp lại), độ đúng (thông qua xác định hiệu suất thu hồi trên nền mẫu thực), xác định giới hạn phát hiện (LOD) và giới hạn định lượng (LOQ).

Xây dựng đường chuẩn: đường chuẩn 5 điểm xác định polyphenol bằng phương pháp UV-Vis được xây dựng trong khoảng 10 ppm 50 ppm. Mỗi điểm được đo lặp lại ba lần, lấy giá trị độ hấp thụ quang trung bình. Kết quả cho phương trình đường chuẩn: $\mathrm{Y}=(0,0083 \pm$ $0,009801)+(0,01003 \pm 0,000297) X$, với hệ số tương quan tốt $\mathrm{R}^{2}=0,99987$.

Độ chụm: độ chụm được đánh giá trên hai nền mẫu chè khô và trà túi lọc, mỗi nền mẫu tiến hành phân tích lặp lại 6 lần. Kết quả độ lặp lại thu được với hai mẫu đều đạt giá trị độ lệch chuẩn tương đối (RSD) là $1,6 \%$, đáp ứng yêu cầu của Hiệp hội các nhà phân tích (AOAC).

Độ đúng: độ đúng được đánh giá trên nền mẫu chè khô và trà túi lọc với ba mức thêm chuẩn ở các nồng độ khác nhau (mức thấp, trung bình và mức cao), tại mỗi mức nồng độ tiến hành phân tích lặp 3 lần để đánh giá độ thu hồi. Kết quả thu được hiệu suất thu hồi đạt trong khoảng 97-103\%, đáp ứng yêu cầu của $\mathrm{AOAC}$.

Xác định LOD, LOQ: được xác định dựa vào giá trị $3 \sigma$ và $10 \sigma$ tương ứng, thực hiện trên nền mẫu trà túi lọc có hàm lượng polyphenol tổng thấp. Các thí nghiệm được phân tích lặp lại 10 lần. Kết quả thu được giá trị LOD là $0,12 \mathrm{mg} / \mathrm{g}$ và $L O Q$ là $0,40 \mathrm{mg} / \mathrm{g}$.

\subsection{Nghiên cứu xác định hàm lượng EGCG trong chè bằng phương pháp HPLC}

\subsubsection{Khảo sát các điều kiện phân tích tối uu}

Tham khảo TCVN 9745-2:2013 [3] và khảo sát sơ bộ trên thiết bị HPLC-PDA, chúng tôi lựa chọn pha động gồm, kênh A: acetonitrile $9 \%+$ acid acetic 2\% + EDTA $20 \mu \mathrm{g} / \mathrm{mL}$, kênh B: acetonitrile $80 \%+$ acid acetic $2 \%+$ EDTA 20 $\mu \mathrm{g} / \mathrm{mL}$, tốc độ dòng $1,0 \mathrm{~mL}$. Chương trình gradient: $100 \%$ pha động kênh A trong 10 phút, sau đó $68 \%$ pha động kênh $A+32 \%$ pha động kênh B trong 10 phút, cuối cùng trở lại $100 \%$ pha động kênh A trước khi bơm mẫu tiếp theo.

Với các điều kiện phân tích này, bước sóng cực đại của EGCG là $275 \mathrm{~nm}$ và thời gian lưu là 
10,5 phút. Kết quả khảo sát với mẫu chuẩn, mẫu chè khô và mẫu chè khô thêm chuẩn đều cho các tín hiệu thời gian lưu ở 10,5 phút với bước sóng cực đại $275 \mathrm{~nm}$, cho thấy phương pháp có độ đặc hiệu tốt.

\subsubsection{Khảo sát dung môi chiết, thời gian và nhiệt độ chiết}

Tương tự polyphenol, tỉ lệ dung môi, nhiệt độ và thời gian chiết EGCG cũng được khảo sát nhằm thu được tối đa hàm lượng EGCG trong mẫu. Kết quả khảo sát các dung môi chiết theo các tỉ lệ methanol: nước là $60: 40,70: 30,80: 20$ và 100\% methanol (Hình 3) cho thấy, khi tăng tỉ lệ methanol thì hàm lượng EGCG cũng tăng. Với tỉ lệ $100 \%$ methanol cho hàm lượng EGCG cao hơn đáng kể so với các tỉ lệ khác. Do đó, dung môi $100 \%$ methanol được lựa chọn là dung môi chiết EGCG trong mẫu chè.

Kết quả khảo sát các thời gian chiết từ 20

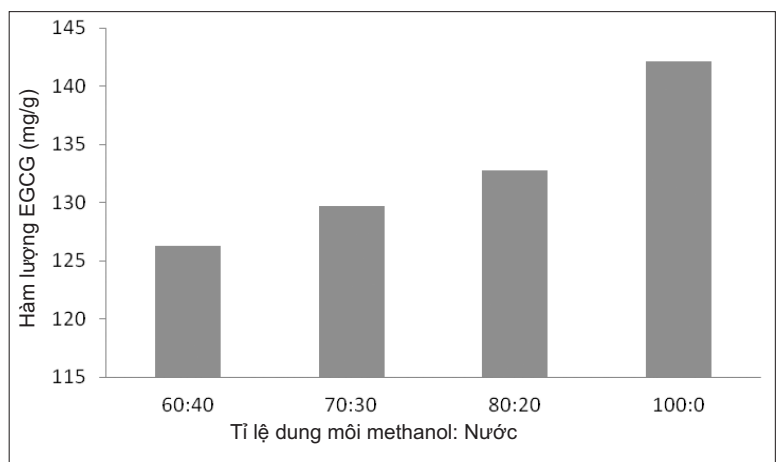

Hinh 3. Khảo sát tỉ lệ dung môi chiết EGCG trong mẫu chè

đến 80 phút và nhiệt độ chiết trong khoảng từ $50^{\circ} \mathrm{C}$ đến $80^{\circ} \mathrm{C}$ cho kết quả tối ưu ở thời gian chiết là 30 phút và nhiệt độ chiết là $70^{\circ} \mathrm{C}$.

\subsection{3.Đánh giá phuoong pháp HPLC xác định} EGCG

Tương tự phương pháp UV-Vis xác định polyphenol, phương pháp HPLC xác định EGCG cũng được đánh giá với các thông số gồm: xây dựng đường chuẩn, độ chụm (thông qua độ lặp lại), độ đúng (thông qua xác định hiệu suất thu hồi trên nền mẫu thực), xác định giới hạn phát hiện (LOD) và giới hạn định lượng (LOQ).

Đường chuẩn ( 5 điểm) xác định $\mathrm{EGCG}$ bằng phương pháp HPLC được xây dựng trong khoảng $1,28 \mathrm{ppm} \div 51,2 \mathrm{ppm}$ cho kết quả phương trình hồi qui: $\mathrm{Y}=(-4611,60 \pm 3616,248)+(15516,36$ \pm 140,1989)X, với hệ số tương quan tốt
$\mathrm{R}^{2}=0,99999$.

Độ lặp lại được đánh giá trên nền mẫu chè khô, phân tích lặp lại 6 lần. Độ lệch chuẩn tương đối thu được là $1,2 \%$, đáp ứng yêu cầu của Hiệp hội các nhà phân tích (AOAC).

Độ thu hồi được đánh giá trên nền mẫu chè khô với ba mức thêm chuẩn ở mức thấp, trung bình và mức cao, mỗi mức nồng độ tiến hành phân tích lặp 3 lần. Kết quả thu được hiệu suất thu hồi đạt trong khoảng $98 \div 102 \%$, đáp ứng yêu cầu của AOAC.

Trên cơ sở qui trình tối ưu, $\mathrm{LOD}$ và $\mathrm{LOQ}$ được xác định theo qui tắc $3 \sigma$ và $10 \sigma$, thực hiện trên nền mẫu chè có hàm lượng EGCG thấp. Các thí nghiệm được phân tích lặp lại 10 lần. Kết quả thu được giá trị $L O D$ là $0,064 \mathrm{mg} / \mathrm{g}$ và $\mathrm{LOQ}$ là $0,213 \mathrm{mg} / \mathrm{g}$.

\subsection{Kết quả phân tích hàm lượng EGCG và polyphenol tổng trong mẫu chè}

Các mẫu chè và sản phẩm chè được mua ngẫu nhiên trên thị trường Hà Nội. Thông tin mẫu cùng kết quả hàm lượng EGCG phân tích bằng phương pháp UV-Vis và polyphenol phân tích bằng phương pháp HPLC được nêu trong bảng 1 và sắc đồ phân tích hàm lượng EGCG trong mẫu chè khô được minh họa trên hình 4 .

Bảng 1. Kết quả phân tích hàm lương polyphenol và EGCG trong các mầu chè

Kết quả thu được cho thấy, các mẫu chè

\begin{tabular}{|c|c|c|}
\hline Tên mẫu & Hàm luợng $\mathbf{E G C G}(\boldsymbol{m g} / \mathbf{g})$ & Hàm luợng tổng polyphenol $(\boldsymbol{m g} / \mathbf{g})$ \\
\hline Chè khô & $32,4 \pm 0,4$ & $183,5 \pm 2,9$ \\
\hline Trà vối & $17,2 \pm 0,2$ & $50,1 \pm 0,8$ \\
\hline Trà túi lọc 1 & $26,0 \pm 0,3$ & $68,9 \pm 1,1$ \\
\hline Trà túi lọc 2 & $16,0 \pm 0,2$ & $35,5 \pm 0,6$ \\
\hline Trà túi lọc 3 & $15,2 \pm 0,2$ & $32,8 \pm 0,5$ \\
\hline Chè nhập khầu 1 & $159,5 \pm 1,9$ & $298,2 \pm 4,7$ \\
\hline Chè nhập khầu 2 & $134,0 \pm 1,6$ & $276,5 \pm 4,4$ \\
\hline Chè nhập khẩu 3 & $147,6 \pm 1,8$ & $282,4 \pm 4,5$ \\
\hline Chè nhập khầu 4 & $148,1 \pm 1,8$ & $286,9 \pm 4,6$ \\
\hline Chè nhập khẩu 5 & $160,3 \pm 1,9$ & $304,5 \pm 4,9$ \\
\hline
\end{tabular}

nhập khẩu và chè khô có hàm lượng polyphenol cao từ $183,5 \div 304,5 \mathrm{mg} / \mathrm{g}$. Trong khi đó, các mẫu trà túi lọc và trà vối có hàm lượng polyphenol thấp hơn, chỉ từ $32,8 \div 68,9 \mathrm{mg} / \mathrm{g}$, có thể do các loại trà này trải qua nhiều công đoạn xử lý, chế biến nên hàm lượng polyphenol bị mất, giảm đi. Tuy nhiên, các mẫu chè khô và trà túi lọc này đều đạt yêu cầu chất lượng so với TCVN (với mẫu chè khô hàm lượng polyphenol $\geq 11 \%$ khối lượng chè, với mẫu trà túi lọc hàm lượng

Tạp chí KIỂM NGHIỆM VÀ AN TOÀN THỤC PHẨM (Số1-2018) | ll 


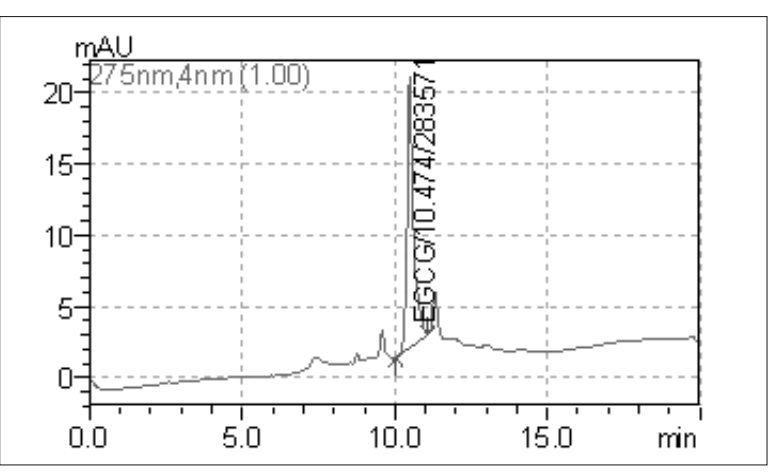

Hinh 4. Sắc đồ phân tích EGCG trong mẫu chè khô

polyphenol $\geq 2 \%$ khối lượng chè) [1].

Kết quả hàm lượng EGCG trong các mẫu chè cũng rất khác nhau, dao động trong khoảng $15,2 \div 160,3 \mathrm{mg} / \mathrm{g}$. Hàm lượng EGCG trong mẫu chè khô nhập khẩu $(134,0 \div 160,3 \mathrm{mg} / \mathrm{g})$ cao hơn nhiều so với trong chè khô, trà vối và trà túi lọc $(15,2 \div 32,4 \mathrm{mg} / \mathrm{g})$, trong đó chè khô có hàm lượng EGCG cao hơn so với trà túi lọc.

Cũng từ các kết quả này cho thấy có mối tương quan giữa hàm lượng $\mathrm{EGCG}$ và hàm lượng polyphenol tổng khi hàm lượng polyphenol tổng cao thì hàm lượng EGCG cũng cao. Do đó, có thể dùng các chỉ tiêu này để đánh giá chất lượng chè. Tuy nhiên, cần có thêm nhiều nghiên cứu hơn nữa để đưa ra được kết luận chính xác hơn.

\section{KẾT LUẦN}

Phương pháp UV-Vis và HPLC đã được nghiên cứu áp dụng nhằm xác định hàm lượng polyphenol tồng và EGCG tương ứng trong các mẫu chè và sản phẩm từ chè. Kết quả phân tích một số mẫu chè cho thấy có mối liên hệ nhất định giữa hàm lượng polyphenol tổng và EGCG, từ đó có thể đề xuất sử dụng các chỉ tiêu này để đánh giá chất lượng chè. Cũng từ kết quả nghiên cứu cho thấy, phương pháp UV-Vis phù hợp để xác định tổng hàm lượng polyphenol và phương pháp HPLC phù hợp để xác định hàm lượng EGCG và có thể mở rộng ra đối với các chất riêng biệt trong các hợp chất polyphenol. Nghiên cứu thực hiện với hi vọng đóng góp một phần nhỏ bé vào việc phát triển quy trình phân tích hàm lượng polyphenol, từ đó giúp kiểm soát chất lượng các sản phẩm chè (trà) trên thị trường, bảo vệ quyền lợi người tiêu dùng.

\section{TÀI LIÊU THAM KHẢO}

1. TCVN 9740 (2013), Tiêu chuẩn quốc gia về Chè xanh Định nghĩa và các yêu cầu cơ bản.

2. TCVN 9745-1 (2013), Chè - xác định các chất đặc trưng của chè xanh và chè đen, phần 1 : Hàm lượng polyphenol tổng số trong chè - Phương pháp đo màu dùng thuốc thử Folin- Ciocalteu.

3. TCVN9745-2 (2013), Chè - xác định các chất đặc trưng của chè xanh và chè đen, phần 2 : Hàm lượng catechin trong chè xanh-Phương pháp sắc kí lỏng hiệu quả cao.

4. Trung tâm nghiên cứu và phát triển công nghệ hóa sinh (2010),"Nghiên cứu sử dụng các hợp chất polyphenol trong các giống chè ở Việt Nam", Hà Nội.

5. Chi-Tang Ho, Jen-Kun Lin, Fereidoon. S (2008), “Tea and Tea Product: Chemistry and Health - Promoting Properties", Nutraceutical science and technology, CRC Press.

6. Marina Naldi, Jessica Fiori, Roberto. G, Aurélie. P, JeanLuc. V, Davy. G, Vincenza. A (2013), "UHPLC determi nation of catechins for the quality control of green tea", Journal of Pharmaceutical and Biomedical Analysis, 88, pp.307-314.

Summary

DETERMINATION OF POLYPHENOL AND EGCG IN TEA AND TEA PRODUCTS BY UV-VIS AND HPLC METHODS

Pham Thi Ngoc Mai', Le Thai Binh', Pham Huy Dong', Nguyen Thi Tuyet Nhung ${ }^{1}$, Pham Tien Duc ${ }^{l}$, Nguyen Thi Anh Huong ${ }^{1 *}$

${ }^{1}$ Faulty of Chemistry, VNU university of Science, Vietnam National University-Hanoi

${ }^{2}$ Vietnam Institute of Industrial Chemistry

Tea and tea products have been known and used for long and became a tradional culture in many countries in the world, including Vietnam. The quality of tea depends on the concentrations of typical active substances such as polyphenol groups and their active substances (EGCG, ECG, catechin,...). Therefore, study of analytical methods to determine these active substances is necessary to evaluate the quality of tea and tea products. In this study, UV-Vis method was selected to determine total polyphenol and HPLC method was selected to determine the main active ingredient of polyphenol group EGCG. The limit of detections are $0.12 \mathrm{mg} / \mathrm{g}$ and 0.064 $\mathrm{mg} / \mathrm{g}$ for total polyphenol and $E G C G$, respectively. These methods were applied to determine the concentrations of total phenol and EGCG in 10 samples of tea and tea products. Results show that the concentrations of total phenol and EGCG vary significantly among samples, but there is a certain relationship between the concentration of total phenol and EGCG in each sample.

Key words: polyphenol, EGCG, tea, UV-Vis, HPLC 